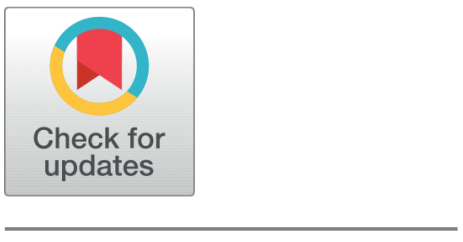

OPEN ACCESS

Received: 12.07.2021

Accepted: 23.10 .2021

Published: 29.11.2021

Citation: Bharathi T, Leo S (2021) A Study on Plithogenic Product Fuzzy Graphs with Application in Social network. Indian Journal of Science and Technology 14(40): 3051-3063. https://doi.org/ 10.17485/IJST/V14i40.1298

* Corresponding author. sleoleo@gmail.com

Funding: None

Competing Interests: None

Copyright: @ 2021 Bharathi \& Leo. This is an open access article distributed under the terms of the Creative Commons Attribution License, which permits unrestricted use, distribution, and reproduction in any medium, provided the original author and source are credited.

Published By Indian Society for Education and Environment (iSee)

ISSN

Print: 0974-6846

Electronic: 0974-5645

\section{A Study on Plithogenic Product Fuzzy Graphs with Application in Social network}

\author{
T Bharathi ${ }^{1}$, S Leo ${ }^{2 *}$ \\ 1 Assistant Professor, Department of Mathematics, Loyola College, University of Madras, \\ Chennai, 600 034, Tamil Nadu, India \\ 2 Research Scholar, Department of Mathematics, Loyola College, University of Madras, \\ Chennai, 600 034, Tamil Nadu, India
}

\section{Abstract}

Objectives: The main objective of this study is to define Plithogenic product fuzzy graphs (PPFGs), and introduce its properties. Method: PPFGs is newly introduced as a new graphical model where P-vertices are characterized by four or more attributes and the attribute values of P-edges are computed using the product operator. Findings: Theoretical discussions and results related to PPFGs, and subgraphs, paths, cycles, trees, bridge and cut vertex in PPFGs are demonstrated with examples. A social network model based on the notion of PPFGs has been presented and analyzed to show the utility and the advantage of Plithogenic product fuzzy graph model. Novelty: Strong and weak P-vertices, and highly strong, strong and weak P-edges are identified to analyze the strength of connectivity between different units. P-order, P-size, P-vertex range, P-edge range, degree, total degree, and average P-weight of P-vertices are computed to examine proximity, significance and centrality of units.

Keywords: Plithogenic fuzzy sets; Fuzzy graphs; Plithogenic fuzzy graphs; Plithogenic product fuzzy graphs; Social networks

\section{Introduction}

Fuzzy sets and fuzzy relations were formulated by L.A. Zadeh in $1965^{(1)}$. The introduction to Fuzzy graphs (FGs) was done by Kaufmann in $1973^{(2)}$. It was further developed by Azriel Rosenfeld in $1975^{(3)}$. Since the single values of membership degree given to vertices and edges from $[0,1]$ in FGs provide limited knowledge and perception regarding any real-life problem under consideration, Intuitionistic fuzzy sets and fuzzy logic, and Intuitionistic fuzzy graphs (IFGs) were introduced by Atanassov in $1986^{(4)}$, and Karunambigai and Parvathi in $2006^{(5)}$ respectively. In IFGs, membership and nonmembership degrees from $[0,1]$ are given to each element with the condition that the sum of membership and non-membership degrees is less than or equal to one. But then there are many real-life situations where the concept of neutrality degree is existent. So, IFGs are insufficient to handle such scenarios.

Cen Zuo et al. in $2019^{(6)}$ introduced Picture fuzzy graphs which is an extension of FGs and IFGs. Picture fuzzy graphs allows the idea of membership, neutral membership and non-membership degrees of an element from $[0,1]$ with the condition that their sum must be less than or equal to one. There are also situations where the models of Product 
vague graphs and Neutrosphic graphs are used to study any vague and inconsistent circumstances ${ }^{(7-9)}$. Neutrosophic sets and Neutrosophic graphs were introduced by Smarandache in 2006 and 2015 respectively ${ }^{(10-12)}$. In Neutrosophic fuzzy graphs membership, indeterminacy and non-membership values, which are within the real standard or nonstandard unit interval ]$^{-} 0,1^{+}$[are allotted to vertices and corresponding edges. In order to apply neutrosophic logic in real-life situations the concept of Single valued neutrosophic graphs (SVNGs) was introduced in 2016 by Broumi et al. ${ }^{(13)}$. In a Single valued neutrosophic graph, membership, indeterminacy and non-membership values of an element are independent entities from $[0,1]$ unlike FGs, IFGs, and other extensions of FGs.

The interesting development in the field of applied mathematics is the emergence of Plithogenic logic and Plithogenic sets by Smarandache in $2017^{(14)}$. The elements belonging to a Plithogenic set are characterized by one or more attributes with the corresponding attribute values. Plithogenic graphs (PGs) were introduced by F. Smarandache et al. in $2020^{(15)}$. PGs is a generalization of Crisp graphs, FGs, IFGs and SVNGs. One of the types of Plithogenic fuzzy graphs (PFGs) is PPFGs which is stated as Type III PFGs in the examples given for PFGs by Smarandache et al. ${ }^{(15)}$. In PPFGs, vertices and edges are characterized by four or more attributes which have their corresponding attribute values from $[0,1]$. The computation of the attribute values of an edge in a PPFG is done using the usual product operator.

Being motivated by the discussions on Plithogenic fuzzy graphs and their applications in ${ }^{(15)}$, the study on PPFGs is undertaken hereby to investigate for newer results and proofs. Since the study on PPFGs is still blank in the literature, we have newly defined PPFGs and introduced some interesting graphical terms in PPFGs with properties, results and examples in section 3, after some relevant definitions in the preliminaries in section 2. Subgraphs, paths, cycles, trees, bridge and cut vertex in PPFGs are discussed in section 4. Since parameters such as degrees, nature of edges, order and size are interesting and important concepts in fuzzy graphs ${ }^{(16-21)}$, we are motivated to define and discuss about P-order, P-size, P-vertex range, P-edge range, degree of a P-vertex, total degree of a P-vertex, minimum degree, maximum degree and nature of P-edges in PPFGs in sections 5 and 6 .

As the number of attributes for a PPFG is greater than or equal to four, it is more reliable and realistic representation of vagueness in certain real-life situations. It is also more efficient in obtaining higher precision in our perception of uncertain scenarios in the decision-making process. PPFG model can be utilized to represent and analyze social network of persons, medical diagnosis of patients, students' performance, resource/computer networking, psychological and sociological issues, weather forecast, etc. where the units involved are to be characterized with many attributes, and the relations between them need to be analyzed depending on the corresponding attribute values allotted to them. Since social networks play a vital role in human life, in section 7 of this paper, we have applied the PPFG model to analyze a small-sized social network.

\section{Preliminaries}

In this section we mainly recall definitions of FGs, degrees of a vertex, order and size of a fuzzy graph, Product fuzzy graphs, Plithogenic fuzzy sets (PFSs) and PFGs which are relevant to the study on PPFGs.

2.1. Definition ${ }^{(2)}$ A fuzzy graph $G:(\alpha, \beta)$ is a pair of functions $\alpha: V \rightarrow[0,1]$ and $\beta: V \times V \rightarrow[0,1]$, such that $\beta(u, v) \leq$ $\alpha(u) \wedge \alpha(v)$ for all $u, v \in V$ where $V$ is the vertex set.

2.2. Definition ${ }^{(16,17)}$ Let $G:(V, \alpha, \beta)$ be a fuzzy graph. Then the degree of a vertex v is defined by $d(v)=\sum_{u \neq v} \beta(v, u)$. The minimum degree of $\mathrm{G}$ is defined by $\delta(G)=\wedge\{d(v) / v \in V\}$. The maximum degree of $\mathrm{G}$ is defined by $\triangle(G)=v\{d(v) / v \in V\}$. The total degree of $v \in V$ is defined by $t d(v)=\sum_{v \neq u} \beta(v, u)+\alpha(v)=d(v)+\alpha(v)$.

2.3. Definition ${ }^{(17)}$ Let $G:(V, \alpha, \beta)$ be a fuzzy graph. Then the order of $\mathrm{G}$ is defined by $O(G)=\sum_{v \in V} \alpha(v)$. The size of $\mathrm{G}$ is defined by $S(G)=\sum_{u \neq v} \beta(u, v)$.

2.4. Definition ${ }^{(22)}$ Let $\mathrm{G}^{*}:(V, E)$ be a graph. $\gamma$ be a fuzzy subset of $V$, and $\tau$ be a fuzzy subset of $V \times V$. We call $G:(\gamma, \tau)$ a product fuzzy graph if $\tau(x, y) \leq \gamma(x) \times \gamma(y)$ for all $x, y \in V$.

2.5. Definition ${ }^{(15)}$ A Plithogenic fuzzy set $U$ is defined as a set where each element is characterized by many attribute values. That is if $c_{1}, c_{2}, \ldots \ldots \ldots, c_{n}$ are $n$ attributes and $X_{1}, X_{2}, \ldots, X_{t} \in U$, then $X_{1}$ 's fuzzy degrees of these attributes is $X_{1}\left(d_{11}, d_{12} \ldots, d_{1 n}\right)$ where $d_{1 i} \in[0,1] ; 1 \leq i \leq n$. Similarly, $X_{2}$ 's fuzzy degrees of these attributes is $X_{2}\left(d_{21}, d_{22} \ldots, d_{2 n}\right)$, where $d_{2 i} \in[0,1] ; 1 \leq i \leq n$. Similary fuzzy degree values for any $X_{k} ; 1 \leq k \leq t$ are assigned. Such a set is called a Plithogenic fuzzy set (PFS). Note that a PFS, in general, may have elements characterized by four or more attributes, which have the corresponding attribute values from $[0,1]$.

2.6. Definition ${ }^{(15)}$ If $U$ is the universal set of discourse and $c_{1}, c_{2}, \ldots, c_{n}$ are the $\mathrm{n}$ attributes and if $X_{1}, X_{2}, \ldots, X_{m} \in U$, then the PFS is given by $X_{1}\left(d_{11}, d_{12} \ldots, d_{1 n}\right), X_{2}\left(d_{21}, d_{22} \ldots, d_{2 n}\right)$, and $X_{m}\left(d_{m 1}, d_{m 2} \ldots, d_{m n}\right)$ where $d_{i j} \in[0,1] ; 1 \leq i \leq m$ and $1 \leq j \leq n$. Thus we can have a graph with vertex set $V_{i}=X_{i}\left(\mathrm{~d}_{i 1}, \mathrm{~d}_{i 2}, \ldots, \mathrm{d}_{i n}\right), 1 \leq i \leq m$. Depending on the problem and the experts opinion, the vertices in $V=\left\{V_{1}, V_{2}, \ldots, V_{m}\right\}$ can be adjacent or not. We call such graphs as PFGs. The concerned edges 
are given values as per the choice of the operator by the researcher. Note that PFGs are distinctly different from FGs. Throughout this paper, in general, we denote a Plithogenic fuzzy graph (PFG) by $G_{F}=\left(V_{F}, E_{F}\right)$, where $V_{F}$ and $E_{F}$ are PFSs of vertices and relevant edges respectively.

\section{Methodology: Plithogenic product fuzzy graphs}

PPFGs has been defined in this section. Basic concepts and properties of PPFGs are newly introduced with examples and results.

3.1. Definition Let $\mathrm{G}_{F}=\left(\mathrm{V}_{F}, \mathrm{E}_{F}\right)$ be a PFG, where $\mathrm{V}_{F}=\left\{\mathrm{V}_{1}, \mathrm{~V}_{2}, \ldots, \mathrm{V}_{n}\right\}$ and $\mathrm{E}_{F}=\left\{\mathrm{E}_{1}, \mathrm{E}_{2}, \ldots, \mathrm{E}_{m}\right\}$ are PFSs of vertices and relevant edges respectively characterized by $\mathrm{k}$ attributes. $\mathrm{G}_{F}$ is said to be a Plithogenic product fuzzy graph (PPFG) denoted by $\mathrm{G}_{p}=\left(\mathrm{V}_{p}, \mathrm{E}_{p}\right)$, if for any two adjacent $\mathrm{V}_{i}, \mathrm{~V}_{j} \in \mathrm{V}_{F}, \mathrm{i} \neq \mathrm{j}$, with the corresponding edge $\mathrm{E}_{c} \in \mathrm{E}_{F}$,

$\mathrm{V}_{i}=\mathrm{X}_{i}\left(\mathrm{~s}_{1}, \mathrm{~s}_{2}, \ldots, \mathrm{s}_{k}\right)$

$\mathrm{V}_{j}=\mathrm{X}_{j}\left(\mathrm{t}_{1}, \mathrm{t}_{2}, \ldots, \mathrm{t}_{k}\right)$

$\mathrm{E}_{c}=\mathrm{Y}_{c}\left(\mathrm{c}_{1}, \mathrm{c}_{2}, \ldots, \mathrm{c}_{k}\right)$ where for any $\mathrm{c}_{d} \in \mathrm{Y}_{c}, \mathrm{c}_{d}=\mathrm{s}_{a} \mathrm{t}_{b}$ with $\mathrm{s}_{a} \in \mathrm{X}_{i}$ and $\mathrm{t}_{b} \in \mathrm{X}_{j} ; \mathrm{d}=\mathrm{a}=\mathrm{b} ; 1 \leq \mathrm{d}, \mathrm{a}, \mathrm{b} \leq \mathrm{k} ; \mathrm{c}_{d}, \mathrm{~s}_{a}, \mathrm{t}_{b} \in[0,1]$. Here $\mathrm{X}_{i}, \mathrm{X}_{j}$ and $\mathrm{Y}_{c}$ are sets of attribute values from $[0,1]$ characterizing $\mathrm{V}_{i}, \mathrm{~V}_{j}$ and $\mathrm{E}_{c}$ respectively in $\mathrm{G}_{F}$.

3.1.1. Note In this study, PPFGs which are undirected and simple (no loop or multiple edges) are considered. Since PFSs, in general, have elements characterized by four or more attributes which have the corresponding attribute values from [0,1], throughout this paper we consider $k \geq 4$ where $k$ is the number of attributes which is the same for any $V \in V_{P}$ and $E \in E_{P}$ in $G_{P}=\left(V_{P}, E_{P}\right)$, where $V_{P}=\left\{V_{1}, V_{2}, \ldots, V_{n}\right\}$ and $E_{P}=\left\{E_{1}, E_{2}, \ldots, E_{m}\right\}$ are PFSs of vertices and relevant edges respectively.

3.1.2. Note In definition 3.1. if $s_{a}$ and $t_{b}$ are from $(0,1)$ with $k$-decimal places and l-decimal places respectively, then $c_{d}$ is with $(k+l)$ decimal places from $(0,1)$.

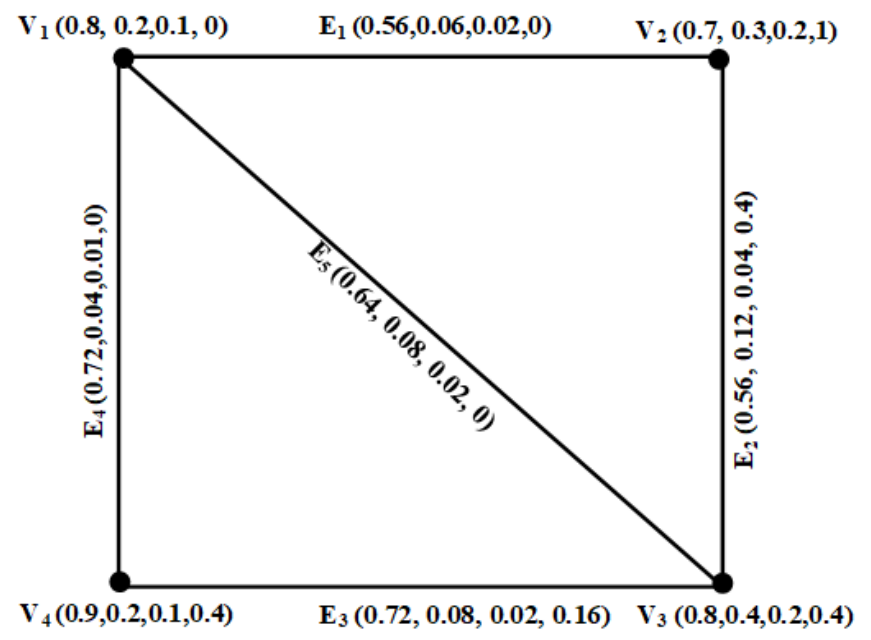

Fig 1. Plithogenic product fuzzy graph

3.2. Definition A vertex of $G_{P}$ is referred as a $P$-vertex. An edge of $G_{P}$ is referred as a $P$-edge. A $P$-vertex in $G_{P}$ is called a $P$-isolated vertex if no $P$-edge is incident to it. The $\mathrm{V}_{P}$-cardinality of $G_{P}$ is the number of $P$-vertices present in it. It is denoted by $\left|V_{p}\left(G_{p}\right)\right|$. The $\mathrm{E}_{P}$-cardinality of $G_{P}$ is the number of $P$-edges present in it. It is denoted by $\left|E_{p}\left(G_{p}\right)\right|$. The sum of the attribute values of any $V \in V_{P}$ is known as its $P$-weight. It is denoted by $P_{V W}(V)$. The sum of the attribute values of any $E \in E_{P}$ is known as its $P$-weight. It is denoted by $P_{E W}(E)$. Note that $0 \leq P_{V W}(V) \leq k$ and $0 \leq P_{E W}(E) \leq k$. Any two P-vertices $V_{i}$ and $V_{j}$ are said to be neighbours if they are adjacent and $P_{E W}\left(V_{i} V_{j}\right)>0$. A P-vertex in a PPFG having exactly one neighbour is called a pendant P-vertex. Otherwise, it is called non-pendent P-vertex. A P-edge incident to a pendant P-vertex is called a pendant P-edge. Otherwise, it is said to be non-pendant P-edge. A P-vertex adjacent to a pendant P-vertex is called an associate of the pendant P-edge.

3.2. Example Let us consider PPFG, $G_{P}=\left(V_{P}, E_{P}\right)$ as in Figure 1 . By computation we have the $\mathrm{V}_{P^{-}}$-cardinality of $G_{P}$, $\mathrm{E}_{P^{-}}$ cardinality of $G_{P}, P$-weight of each $P$-vertex and $P$-weight of each $P$-edge as follows:

$\left|V_{P}\left(G_{P}\right)\right|=4 ;\left|E_{P}\left(G_{P}\right)\right|=5$

$P_{V W}\left(V_{1}\right)=1.1 ; P_{V W}\left(V_{2}\right)=2.2 ; P_{V W}\left(V_{3}\right)=1.8 ; P_{V W}\left(V_{4}\right)=1.6$

$$
P_{E W}\left(E_{1}\right)=0.64 ; P_{E W}\left(E_{2}\right)=1.12 ; P_{E W}\left(E_{3}\right)=0.98 ; P_{E W}\left(E_{4}\right)=0.77 ; P_{E W}\left(E_{5}\right)=0.74
$$


3.3. Proposition In a Plithogenic product fuzzy graph, an attribute value of any P-edge is zero if either of the corresponding attribute values of its adjacent P-vertices or both are zero.

Proof: Let us consider $E_{c} \in E_{P}$ of a PPFG, $G_{P}=\left(V_{P}, E_{P}\right)$ with attribute values $c_{1}, c_{2}, \ldots, c_{k}=0$. Let $V_{i}$, $V_{j} \in V_{P}$ be the adjacent P-vertices to $E_{c}$ with attribute values $s_{1}, s_{2}, \ldots, s_{k}$ and $t_{1}, t_{2}, \ldots, t_{k}$ respectively. i.e., $s_{1}, s_{2}, \ldots, s_{k} \in V_{i}, t_{1}, t_{2}, \ldots, t_{k} \in V_{j}$ and $c_{1}, c_{2}, \ldots, c_{k} \in E_{c}$

Since $c_{d}=s_{a} t_{b}$ for any $c_{d} \in E_{c}$ with $s_{a} \in V_{i}$ and $t_{b} \in V_{j} ; 1 \leq d, a, b \leq k ; d=a=b ; c_{d}, s_{a}, t_{b} \in[0,1]$, it follows that for $c_{d}=0$, either $s_{a}=0$ or $t_{b}=0$, or both $s_{a}=t_{b}=0$. Hence the result.

3.4. Corollary In a Plithogenic product fuzzy graph, $G_{P}=\left(V_{P}, E_{P}\right)$ for any adjacent $V_{i}, V_{j} \in V_{P}$ if $s_{1}, s_{2}, \ldots, s_{k}=1 \in V_{i}$ and $t_{1}, t_{2}, \ldots, t_{k}=1 \in V_{j}$, then $c_{1}, c_{2}, \ldots, c_{k}=1 \in E_{c}$ where $E_{c} \in E_{P}$ is the P-edge between to $V_{i}$ and $V_{j}$.

Proof: Let us consider any two adjacent P-vertices $V_{i}, V_{j} \in V_{P}$ of a PPF, $G_{P}=\left(V_{P}, E_{P}\right)$ with attribute values $s_{1}, s_{2}, \ldots, s_{k}=1$ and $t_{1}, t_{2}, \ldots, t_{k}=1$ respectively. Let $E_{c} \in E_{P}$ be the P-edge between $V_{i}$ and $V_{j}$ with attribute values $c_{1}, c_{2}, \ldots, c_{k}$.

Since $c_{d}=s_{a} t_{b}$ for any $c_{d} \in E_{c}$ with $s_{a} \in V_{i}$ and $t_{b} \in V_{j} ; 1 \leq d, a, b \leq k ; d=a=b ; c_{d}, s_{a}, t_{b} \in[0,1]$, it follows that $c_{1}, c_{2}, \ldots, c_{k}=1 \in E_{c}$ since $s_{1}, s_{2}, \ldots, s_{k}=1 \in V_{i}$ and $t_{1}, t_{2}, \ldots, t_{k}=1 \in V_{j}$. Hence the result.

3.5. Proposition In any Plithogenic product fuzzy graph the P-weight of any P-edge is less than or equal to the minimum of the P-weights of the corresponding adjacent P-vertices.

Proof: Let $G_{P}=\left(V_{P}, E_{P}\right)$ be a PPFG. For any P-edge $E_{c} \in E_{P}$ with the attribute values $c_{1}, c_{2}, \ldots, c_{k}$, let us consider the corresponding adjacent P-vertices $V_{i}, V_{j} \in V_{P}$ with attribute values $s_{1}, s_{2}, \ldots, s_{k}$ and $t_{1}, t_{2}, \ldots, t_{k}$ respectively.

(i.e.) $s_{1}, s_{2}, \ldots, s_{k} \in V_{i}, t_{1}, t_{2}, \ldots, t_{k} \in V_{j}$ and $c_{1}, c_{2}, \ldots, c_{k} \in E_{c}$ where $c_{d}=s_{a} t_{b}$ for any $c_{d} \in E_{c}$ with $s_{a} \in V_{i}$ and $t_{b} \in V_{j} ; d=$ $a=b ; 1 \leq d, a, b \leq k ; c_{d}, s_{a}, t_{b} \in[0,1]$.

We know that $P_{V W}\left(V_{i}\right)=s_{1}+s_{2}+\cdots+s_{k} ; P_{V W}\left(V_{j}\right)=t_{1}+t_{2}+\cdots+t_{k} ;$ and $P_{E W}\left(E_{c}\right)=c_{1}+c_{2}+\cdots+c_{k}$. Then the following cases hold true:

Case (i): When $s_{1}, s_{2}, \ldots, s_{k} \in(0,1)$ and $t_{1}, t_{2}, \ldots, t_{k} \in(0,1)$,

$c_{1}+c_{2}+\cdots+c_{k}<\min \left\{s_{1}+s_{2}+\cdots+s_{k}, t_{1}+t_{2}+\cdots+t_{k}\right\}$

Case (ii): When either $s_{1}, s_{2}, \ldots, s_{k}=0$ or $t_{1}, t_{2}, \ldots, t_{k}=0$, then $c_{1}+c_{2} \cdots+c_{k}=0$ which is the $\min \left\{s_{1}+s_{2}+\cdots+s_{k}, t_{1}+t_{2}+\cdots+t_{k}\right\}$.

Case (iii): when $s_{1}, s_{2}, \ldots, s_{k}=1$ and $t_{1}, t_{2}, \ldots, t_{k}=1$, then $c_{1}, c_{2}, \ldots, c_{k}=1$. (i.e.) if $s_{1}+s_{2}+\cdots+s_{k}=1+1+\ldots k$ times and $t_{1}+t_{2}+\cdots+t_{k}=1+1+\ldots k$ times, then $c_{1}+c_{2}, \cdots+c_{k}=1+1+\ldots k$ times which is $\operatorname{the} \min \left\{s_{1}+s_{2}+\cdots+s_{k}, t_{1}+\right.$ $\left.t_{2}+\cdots+t_{k}\right\}$

Hence the proposition is proved.

3.6. Theorem Every Plithogenic product fuzzy graph is a Plithogenic fuzzy graph, but the converse need not be true.

Proof: Let us assume that $G_{P}=\left(V_{P}, E_{P}\right)$ be a PPFG where $V_{P}$ and $E_{P}$ are PFSs of P-vertices and relevant P-edges respectively in $G_{P}$. We need to prove that, $G_{P}$ is a PFG. For any P-edge $E_{c} \in E_{P}$ with attribute values $c_{1}, c_{2}, \ldots, c_{k}$, let $V_{i}, V_{j} \in V_{P}$ be its adjacent P-vertices with attribute values $s_{1}, s_{2}, \ldots, s_{k}$ and $t_{1}, t_{2}, \ldots, t_{k}$ respectively. This follows that for any $c_{d} \in E_{c}, c_{d}=s_{a} t_{b}$ , where $s_{a} \in V_{i}$ and $t_{b} \in V_{j} ; 1 \leq d, a, b \leq k ; d=a=b ; c_{d}, s_{a}, t_{b} \in[0,1]$. Since the attribute values $c_{1}, c_{2}, \ldots, c_{k}$ of any P-edge $E_{c} \in E_{P}$ are obtained using the usual product operator with the condition that for any $c_{d} \in E_{c}, c_{d}=s_{a} t_{b}$, where $s_{a} \in V_{i}$ and $t_{b} \in V_{j} ; 1 \leq d, a, b \leq k ; d=a=b ; c_{d}, s_{a}, t_{b} \in[0,1]$, this implies that $E_{c} \in E_{F}$ and $V_{i}, V_{j} \in V_{F}$ of PFG, $G_{F}=\left(V_{F}, E_{F}\right)$. i.e., $G_{P}=\left(V_{P}, E_{P}\right)$ equals $G_{F}=\left(V_{F}, E_{F}\right)$. Hence $G_{P}$ is a PFG.

Conversely, let $G_{F}=\left(V_{F}, E_{F}\right)$ be a PFG where $V_{F}$ and $E_{F}$ are PFSs of vertices and relevant edges respectively. For any edge $E_{c} \in E_{F}$ with attribute values $c_{1}, c_{2}, \ldots, c_{k}$, let its adjacent vertices be $V_{i}, V_{j} \in V_{F}$ with attribute values $s_{1}, s_{2}, \ldots, s_{k}$ and $t_{1}, t_{2}, \ldots, t_{k}$ respectively. This follows that for any $c_{d} \in E_{c}$ either $c_{d}=s_{a} t_{b}$ or $c_{d} \neq s_{a} t_{b}$, where $s_{a} \in V_{i}$ and $t_{b} \in V_{j} ; 1 \leq d, a, b \leq$ $k ; d=a=b ; c_{d}, s_{a}, t_{b} \in[0,1]$.

If $c_{d}=s_{a} t_{b}$ for any $c_{d} \in E_{c}$ with $s_{a} \in V_{i}$ and $t_{b} \in V_{j} ; 1 \leq d, a, b \leq k ; d=a=b$, then $E_{c} \in E_{P}$ and its adjacent vertices $V_{i}, V_{j} \in V_{P}$ of $G_{P}=\left(V_{P}, E_{P}\right)$. i.e., $G_{F}=\left(V_{F}, E_{F}\right)$ equals $G_{P}=\left(V_{P}, E_{P}\right)$. Hence $G_{F}$ is a PPFG. If $c_{d} \neq s_{a} t_{b}$ for any $c_{d} \in E_{c}$ with $s_{a} \in V_{i}$ and $t_{b} \in V_{j} ; 1 \leq d, a, b \leq k ; d=a=b$, then $E_{c} \notin E_{P}$ and its adjacent vertices $V_{i}, V_{j} \notin V_{P} o f G_{P}=\left(V_{P}, E_{P}\right)$. i.e., $G_{F}=\left(V_{F}, E_{F}\right)$ is not equal to $G_{P}=\left(V_{P}, E_{P}\right)$. Therefore $G_{F}$ is not a PPFG. Hence the proof.

3.7. Proposition The P-weight of any $\mathrm{P}$-vertex in a Plithogenic product fuzzy graph is always less than or equal to $\mathrm{k}$ which is the number of attributes.

Proof: Let $G_{P}=\left(V_{P}, E_{P}\right)$ be a PPFG where $V_{P}$ and $E_{P}$ are PFSs of P-vertices and relevant P-edges respectively. For any $V \in V_{P}$ with attribute values $s_{1}, s_{2}, \ldots, s_{k}$, the following two cases hold true:

Case (i): When $s_{1}, s_{2}, \ldots, s_{k} \in[0,1)$, then $P_{V W}(V)<k$

Case (ii): When $s_{1}, s_{2}, \ldots, s_{k}=1$, then $P_{V W}(V)=k$. Hence the result.

3.8. Definition Let $G_{P}=\left(V_{P}, E_{P}\right)$ be a PPFG, where $V_{P}=\left\{V_{1}, V_{2}, \ldots, V_{n}\right\}$ and $E_{P}=\left\{E_{1}, E_{2}, \ldots, E_{m}\right\}$ are the PFSs of Pvertices and relevant P-edges respectively. The Supremum $\left(S_{A}\right)$ of any P-vertex $V \in V_{P}$ is the highest attribute value associated 
with $V$ and it is denoted by $S_{A}(V)$. The Supremum $\left(S_{A}\right)$ of any P-edge $E \in E_{P}$ is the highest attribute value of associated with $E$ and it is denoted by $S_{A}(E)$. The Infimum $\left(I_{A}\right)$ of any P-vertex $V \in V_{P}$ is the least attribute value associated with $V$ and it is denoted by $I_{A}(V)$. The Infimum $\left(I_{A}\right)$ of any P-edge $E \in E_{P}$ is the least attribute value of associated with $E$ and it is denoted by $I_{A}(E)$. Any $V \in V_{P}$ is a central P-vertex of $G_{P}$, if it is adjacent to the maximum number of P-vertices in $G_{P}$. It is denoted by $C_{P V}\left(G_{P}\right)$. Note that it is possible to have more than one central P-vertices in a PPFG.

3.8. Example Let us consider a PPFG, $G_{P}=\left(V_{P}, E_{P}\right)$ where $V_{P}=\left\{V_{1}, V_{2}, V_{3}, V_{4}\right\}$ and $E_{P}=\left\{E_{1}, E_{2}, E_{3}, E_{4}\right\}$

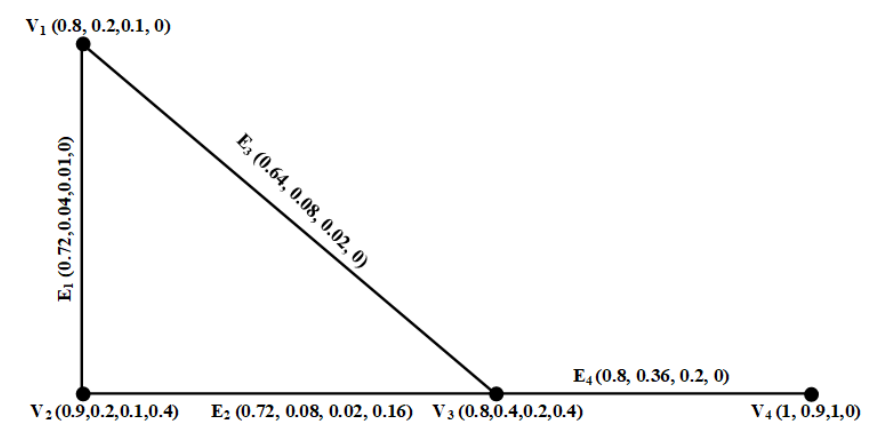

Fig 2. Supremum and Infimum of each P-vertex and each P-edge and Central P-vertex

We have the supremum and the infimum of each P-vertex, the supremum and the infimum of each P-edge, and the central P-vertex of $G_{P}$ as follows:

$$
\begin{gathered}
S_{A}\left(V_{1}\right)=0.8, I_{A}\left(V_{1}\right)=0 ; S_{A}\left(V_{2}\right)=0.9, I_{A}\left(V_{2}\right)=0.1 ; S_{A}\left(V_{3}\right)=0.8, I_{A}\left(V_{3}\right)=0.2 ; \\
S_{A}\left(V_{4}\right)=1, I_{A}\left(V_{4}\right)=0 ; S_{A}\left(E_{1}\right)=0.72, I_{A}\left(E_{1}\right)=0 ; S_{A}\left(E_{2}\right)=0.72, I_{A}\left(E_{2}\right)=0.02 ; \\
S_{A}\left(E_{3}\right)=0.64, I_{A}\left(E_{3}\right)=0 ; S_{A}\left(E_{4}\right)=0.8, I_{A}\left(E_{4}\right)=0 ; C_{P V}\left(G_{P}\right)=V_{3}
\end{gathered}
$$

\section{Subgraphs, Path, Cycle, tree, bridge and cut vertex in PPFGs}

4.1. Definition Let $G_{P}=\left(V_{P}, E_{P}\right)$ be a PPFG. $H_{P}=\left(R_{P}, S_{P}\right)$ is said to be a P-vertex subgraph of $G_{P}$, if it is induced by the elimination of a P-vertex or P-vertices in $G_{P}$. Here $R_{P} \subset V_{P}$ and $S_{P} \subset E_{P} . H_{P}=\left(R_{P}, S_{P}\right)$ is said to be a P-spanning subgraph (Pedge subgraph) of $G_{P}$, if it is induced by the elimination of a P-edge or P-edges in $G_{P}$. Here $R_{P}=V_{P}$ and $S_{P} \subset E_{P} . H_{P}=\left(R_{P}, S_{P}\right)$ is said to be a P-vertex-edge subgraph of $G_{P}$, if it is induced by the removal of P-vertex/P-vertices and P-edge/P-edges in $G_{P}$. Here $R_{P} \subset V_{P}$ and $S_{P} \subset E_{P}$. It is to be noted that a subgraph of PPFG need not be always a connected PPFG.

4.1. Example Consider $G_{P}$ in Fig. 3 as the original PPFG. The P-vertex subgraph of $G_{P}$ in Fig. 4 is obtained by removing $V_{1}, V_{4}, V_{7}, V_{8}$ and $V_{9}$. The P-edge subgraph of $G_{P}$ in Fig. 5 is obtained by the removal of the P-edges $E_{6}, E_{7}, E_{11}$ and $E_{12}$. Similarly the P-vertex-edge subgraph in Fig. 6 is obtained by the elimination of $V_{7}$ and $E_{1}, E_{2}, E_{5}, E_{12}$.

Table 1. P-weights of P-vertices of $G_{P}$

\begin{tabular}{llllllllll}
\hline P-vertices & $V_{1}$ & $V_{2}$ & $V_{3}$ & $V_{4}$ & $V_{5}$ & $V_{6}$ & $V_{7}$ & $V_{8}$ & $V_{9}$ \\
\hline$P_{V W}$ & 2 & 1 & 1.4 & 2.7 & 1.4 & 1.3 & 1.7 & 2.3 & 1.7 \\
\hline
\end{tabular}

Table 2. P-weights of P-edges of Gp

\begin{tabular}{lllllllllllll}
\hline P-edges & $E_{1}$ & $E_{2}$ & $E_{3}$ & $E_{4}$ & $E_{5}$ & $E_{6}$ & $E_{7}$ & $E_{8}$ & $E_{9}$ & $E_{10}$ & $E_{11}$ & $E_{12}$ \\
\hline$P_{E W}$ & 0.25 & 0.22 & 1.53 & 0.36 & 0.47 & 0.89 & 0.45 & 1.31 & 0.93 & 0.51 & 0.78 & 1.06 \\
\hline
\end{tabular}

4.2. Theorem Every subgraph of a Plithogenic product fuzzy graph is a Plithogenic product fuzzy graph.

Proof : Let $G_{P}=\left(V_{P}, E_{P}\right)$ be a PPFG, where $V_{P}$ and $E_{P}$ are PFSs of P-vertices and relevant P-edges respectively. Consider $H_{P}=\left(R_{P}, S_{P}\right)$ to be a subgraph of $G_{P}$, where $R_{P}$ and $S_{P}$ are PFSs of P-vertices and relevant P-edges respectively in $H_{P}$. 


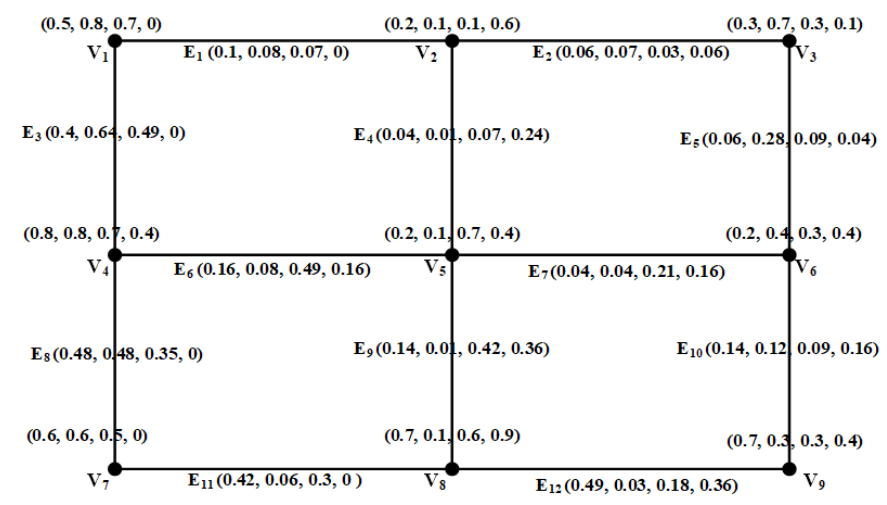

Fig 3. Plithogenic product fuzzy graph, $G_{p}$

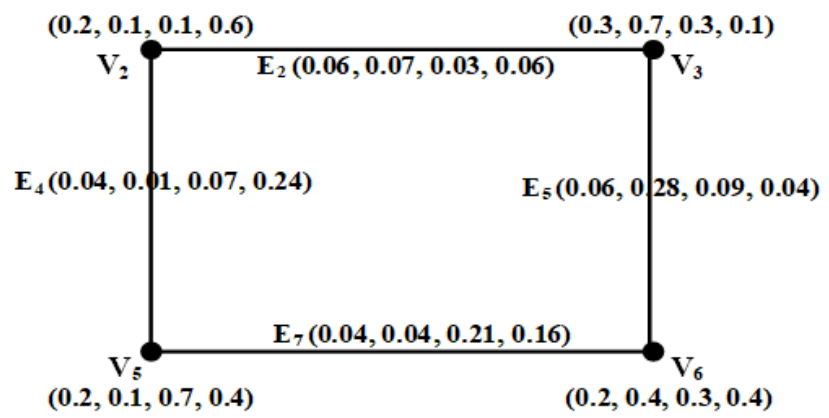

Fig 4. P- vertex subgraph of $\mathrm{Gp}$

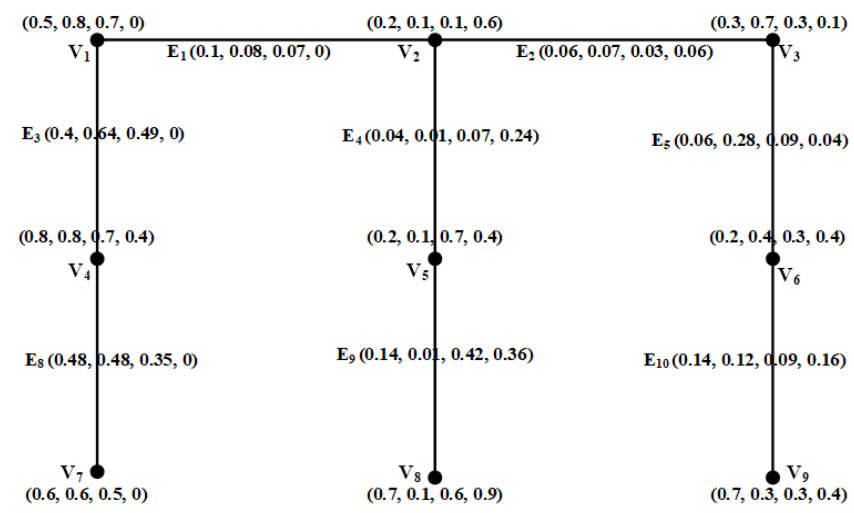

Fig 5. P-edge subgraph of Gp

Case (i): If $H_{P}=\left(R_{P}, S_{P}\right)$ is a P-vertex subgraph, then $R_{P} \subset V_{P}$ and $S_{P} \subset E_{P}$. This implies that every $V \in R_{P}$ is also an element of $V_{P}$ with the same attribute values and, every $E \in S_{P}$ is also an element of $E_{P}$ with the same attribute values. This proves that by definition 3.1. $H_{P}=\left(R_{P}, S_{P}\right)$ is a PPFG.

Case (ii): If $H_{P}=\left(R_{P}, S_{P}\right)$ is a P-edge subgraph, then $R_{P}=V_{P}$ and $S_{P} \subset E_{P}$. This implies that every $V$ in $R_{P}$ is also in $V_{P}$ with the same attribute values and, every $E \in S_{P}$ is also an element of $E_{P}$ with the same attribute values. This proves that by definition 3.1. $H_{P}=\left(R_{P}, S_{P}\right)$ is a PPFG.

Case (iii): If $H_{P}=\left(R_{P}, S_{P}\right)$ is a P-vertex-edge subgraph, then $R_{P} \subset V_{P}$ and $S_{P} \subset E_{P}$. This implies that every $V$ in $R_{P}$ is also an element of $V_{P}$ with the same attribute values. Similarly, every $E \in S_{P}$ is also an element of $E_{P}$ with the same attribute values. This follows that by definition 3.1., $H_{P}=\left(R_{P}, S_{P}\right)$ is a PPFG. Hence the proof. 


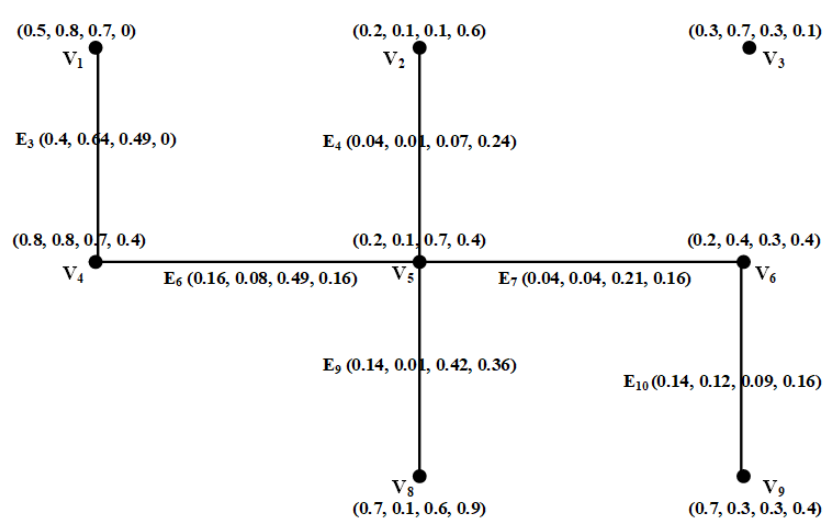

Fig 6. P-vertex-edge subgraph of Gp

4.3. Proposition Every P-vertex subgraph of a connected Plithogenic product fuzzy graph is also P-vertex-edge subgraph.

Proof: Consider $G_{P}=\left(V_{P}, E_{P}\right)$ to be a connected PPFG, where $V_{P}$ and $E_{P}$ are PFSs of P-vertices and relevant P-edges respectively in $G_{P}$. Let $H_{P}=\left(R_{P}, S_{P}\right)$ a P-vertex subgraph of $G_{P}$, where $R_{P}$ and $S_{P}$ are PFSs of P-vertices and relevant P-edges respectively in $H_{P}$. This implies that $R_{P} \subset V_{P}$ and $S_{P} \subset E_{P}$. By definition 4.1., $H_{P}$ is obtained by the elimination of at least one $V \in V_{P}$ and its adjacent P-edges in $G_{P}$. This follows that $H_{P}$ is also P-vertex-edge subgraph of $G_{P}$. Hence the proof.

4.4. Definition A Plithogenic product fuzzy path (PPFP) is a sequence $V_{0}, E_{1}, V_{1}, E_{2}, \ldots, V_{n-1}, E_{n}, V_{n}$ of distinct P-vertices and P-edges such that for any P-edge $E_{i}$ with end points $V_{i-1}$ and $V_{i}, P_{E W}\left(E_{i}\right)>0$ where $1 \leq i \leq n$. Here $\mathrm{n}$ is the length of the PPFP. The strength of a PPFP is defined as $\min \left\{P_{E W}\left(E_{i}\right): i=1,2, \ldots n\right\}$. In other words, the strength of a PPFP is the minimum of the P-weights of all the P-edges in it. A PPFP : $V=V_{0}, E_{1}, V_{1}, E_{2}, \ldots, V_{n-1}, E_{n}, V_{n}=W$ from $\mathrm{V}$ to $\mathrm{W}$ is said to be strong if all the P-vertices in it are strong P-vertices. A PPFP from $\mathrm{V}$ to $\mathrm{W}$ is called a Plithogenic product fuzzy cycle (PPFC) provided $V_{0}=V_{n}$ and $n \geq 3$. A PPFC of odd length is said to be an odd PPFC. Similarly, a PPFC of even length is said to be an even PPFC. If there is no PPFC in a PPFG, then it is said to be acyclic. The strength of connectedness between any two P-vertices $V_{i}$ and $V_{j}$ in a PPFG is defined as the maximum of the strengths of all Plithogenic product fuzzy paths (PPFPs) between $V_{i}$ and $V_{j}$ and it is denoted by $S_{C O N P}\left(V_{i}, V_{j}\right)$. A PPFP that contains $S_{C O N P}\left(V_{i}, V_{j}\right)$ between any two P-vertices $V_{i}$ and $V_{j}$ in a PPFG is called as the strongest PPFP provided it is also a strong PPFP. It is to be noted that there can be more than one strongest PPFPs in a PPFG. A PPFG is said to be connected if every pair of P-vertices in it has at least one PPFP between them. Otherwise, it is said to be a disconnected PPFG. A PPFG which is connected and acyclic is called a Plithogenic product fuzzy tree (PPFT). A P-edge in a PPFG is said to be a bridge if its removal decreases the strength of connectedness between some pair of P-vertices in it. A P-vertex in a PPFG is said to be a cut vertex if its removal decreases the strength of connectedness between some pair of P-vertices in it.

4.4. Example In Fig. $3, V_{1}, E_{1}, V_{2}, E_{2}, V_{3}, E_{5}, V_{6}$ is a PPFP of length 3. Since $P_{E V}\left(E_{1}\right)=0.25, P_{E V}\left(E_{2}\right)=0.22$ and $P_{E V}\left(E_{5}\right)=0.47$, the strength of the path is 0.22 . The other PPFPs between $V_{1}$ and $V_{6}$ are $V_{1}, E_{1}, V_{2}, E_{4}, V_{5}, E_{7}, V_{6}$ whose strength is $0.25 ; V_{1}, E_{3}, V_{4}, E_{6}, V_{5}, E_{7}, V_{6}$ whose strength is $0.45 ; V_{1}, E_{3}, V_{4}, E_{8}, V_{7}, E_{11}, V_{8}, E_{12}, V_{9}, E_{10}, V_{6}$ whose strength is 0.51 and $V_{1}, E_{3}, V_{4}$, $E_{8}, V_{7}, E_{11}, V_{8}, E_{9}, V_{5}, E_{7}, V_{6}$ whose strength is 0.45 . Therefore, strength of connectedness between $V_{1}$ and $V_{6}$ i.e., $S_{C O N P}\left(V_{1}, V_{6}\right)$ is 0.51 which is the maximum of all strengths of the PPFPs between $V_{1}$ and $V_{6}$ in Figure $3 . V_{1}, E_{3}, V_{4}, E_{8}, V_{7}$ and $V_{1}, E_{3}, V_{4}$ and $V_{4}, E_{8}, V_{7}$ and $V_{8}, E_{3}, V_{4}$ are strongest PPFPs in Fig. 3. $E_{12}$ is a bridge, since its removal reduces the strength of connectedness between $V_{1}$ and $V_{6}$ i.e., when $E_{12}$ is removed, then $S_{C O N P}\left(V_{1}, V_{6}\right)=0.45$. Similarly, $V_{9}$ is a cut vertex as its removal reduces the strength of connectedness between $V_{1}$ and $V_{6}$ Fig. 3. The PPFGs given in Fig. 5 and Fig. 6 are Plithogenic product fuzzy trees since there are no Plithogenic product fuzzy cycles in them, while the PPFG in Fig. 4 is a PPFC of length 4.

4.5. Proposition The strength and the strength of connectedness are identical for every Plithogenic product fuzzy path in a Plithogenic product fuzzy tree.

Proof : Let $G_{P}=\left(V_{P}, E_{P}\right)$ be a PPFT, where $V_{P}$ and $E_{P}$ are PFSs of P-vertices and relevant P-edges respectively in $G_{P}$. Let $V_{i}, V_{j} \in V_{P}$ be any two P-vertices in $G_{P}$. Suppose there exists more than one PPFP between $V_{i}$ and $V_{j}$. Then there exists at least one PPFC in $G_{P}$ which passes through either $V_{i}$ or $V_{j}$, or both $V_{i}$ and $V_{j}$, or some points between $V_{i}$ and $V_{j}$, since $G_{P}$ is connected. This contradicts that $G_{P}$ is a PPFT. Therefore, between $V_{i}$ and $V_{j}$ there exists a unique path. This implies that strength and strength of connectedness between $V_{i}$ and $V_{j}$ are identical. Hence the proof. 
4.6. Proposition The longest Plithogenic product fuzzy path in a Plithogenic product fuzzy cycle of length $\mathrm{n}$ is of length $n-1$.

Proof Let $G_{P}=\left(V_{P}, E_{P}\right)$ be a PPFC, where $V_{P}$ and $E_{P}$ are PFSs of P-vertices and relevant P-edges respectively in $G_{P}$ of length n. By definition 4.4., $G_{P}-E$ is a PPFP, where $E \in E_{P}$ is any P-edge in $G_{P}$. Since the length of $G_{P}$ is n, the length of $G_{P}-E$ is $n-1$. Since $G_{P}$ is a PPFC, $G_{P}-E$ is a PPFP of maximum length, i.e., n-1, in $G_{P}$. Hence the proof.

4.7. Definition ${ }^{(15)}$ A PPFG, $G_{P}=\left(V_{P}, E_{P}\right)$ is said to be a complete PPFG if every $V \in V_{P}$ is adjacent with the rest of the P-vertices in $G_{P}$. The PPFG given in Fig. 8 is a complete PPFG.

\section{P-order, P-size, Degree, P-vertex range and P-edge range in Plithogenic product fuzzy graphs}

In this section, $\mathrm{P}$-order, $\mathrm{P}$-size, $\mathrm{P}$-vertex range, $\mathrm{P}$-edge range, degree of a $\mathrm{P}$-vertex, total degree of a $\mathrm{P}$-vertex, the minimum degree and the maximum degree of a PPFG are defined and discussed with examples.

5.1. Definition The P-order of a PPFG, $G_{P}=\left(V_{P}, E_{P}\right)$ is the sum of the P-weights of all the P-vertices in it, and it is denoted by $P_{O}\left(G_{P}\right)$. The P-size of $G_{P}$ is the sum of the P-weights of all the P-edges in it, and it is denoted by $P_{S}\left(G_{P}\right)$. Note that $P_{S}\left(G_{P}\right)$ is always less than $P_{O}\left(G_{P}\right)$ when the attribute values of all elements of $V_{P}$ are from $(0,1)$.

The difference between the maximum P-weight $\left(\triangle_{V W}\right)$ and the minimum P-weight $\left(\delta_{V W}\right)$ of the P-vertices in $G_{P}$ is said to be its P-vertex range and it is denoted by $P_{V R}\left(G_{P}\right)$. The difference between the maximum P-weight $\left(\triangle_{E W}\right)$ and the minimum P-weight $\left(\delta_{E W}\right)$ of the P-edges in $G_{P}$ is said to be its P-edge range and it is denoted by $P_{E R}\left(G_{P}\right)$.

5.2. Definition Let $G_{P}=\left(V_{P}, E_{P}\right)$ be a PPFG, where $V_{P}=\left\{V_{1}, V_{2}, \ldots, V_{n}\right\}$ and $E_{P}=\left\{E_{1}, E_{2}, \ldots, E_{m}\right\}$ are PFSs of P-vertices and P-edges respectively. The degree of any P-vertex $V \in V_{P}$ is the sum of the P-weights of the P-edges incident to $V$ and it is denoted by $d_{P}(V)$. The total degree of any P-vertex $V \in V_{P}$ is the sum of the P-weights of the P-edges incident to $V$ and $P_{V W}(V)$. It is denoted by $t d_{P}(V)$. The minimum degree of $G_{P}=\wedge\left\{d_{P}\left(V_{i}\right): V_{i} \in V_{P} ; i=1,2, \ldots, n\right.$ and it is denoted by $\delta_{P}\left(G_{P}\right)$. The maximum degree of $G_{P}=\vee\left\{d_{P}\left(V_{i}\right): V_{i} \in V_{P} ; i=1,2, \ldots, n\right.$ and it is denoted by $\triangle_{P}\left(G_{P}\right)$.

5.2. Example Consider a PPFG, $G_{P}=\left(V_{P}, E_{P}\right)$ as in Fig. 2. By usual computation, we have P-order, P-size, P-vertex range, P-edge range, degree of each $\mathrm{P}$-vertex, total degree of each P-vertex, the minimum degree and the maximum degree of $G_{P}$ as follows:

$$
\begin{gathered}
P_{V W}\left(V_{1}\right)=1.1 ; P_{V W}\left(V_{2}\right)=1.6 ; P_{V W}\left(V_{3}\right)=1.8 ; P_{V W}\left(V_{4}\right)=2.9 \\
P_{E W}\left(E_{1}\right)=0.77 ; P_{E W}\left(E_{2}\right)=0.98 ; P_{E W}\left(E_{3}\right)=0.74 ; P_{E W}\left(E_{4}\right)=1.36 \\
P_{O}\left(G_{P}\right)=7.4, P_{S}\left(G_{P}\right)=3.85, P_{V R}\left(G_{P}\right)=\triangle_{V W}-\delta_{V W}=1.8 \\
P_{E R}\left(G_{P}\right)=\triangle_{E W}-\delta_{E W}=0.62 \\
d_{P}\left(V_{1}\right)=1.51 ; d_{P}\left(V_{2}\right)=1.75 ; d_{P}\left(V_{3}\right)=3.08 ; d_{P}\left(V_{4}\right)=1.36 \\
\delta_{P}\left(G_{P}\right)=1.36 ; d_{P}\left(V_{1}\right)=2.61 ; t d_{P}\left(V_{2}\right)=3.35 ; t d_{P}\left(V_{3}\right)=4.88 ; t d_{P}\left(V_{4}\right)=4.26 \\
\left.G_{P}\right)=3.08
\end{gathered}
$$

\subsection{Properties of P-vertex range and P-edge range}

- For some $V \in V_{P}$ if $P_{V W}(V)=0$, then $P_{V R}\left(G_{P}\right)=\triangle_{V W}-\delta_{V W}=\triangle_{V W}-0=\triangle_{V W}$

- If some $V_{i}, V_{j} \in V_{P}$ with attribute values $s_{1}, s_{2}, \ldots, s_{k}=1$ and $t_{1}, t_{2}, \ldots, t_{k}=0$ respectively, then $P_{V R}\left(G_{P}\right)=1+1+\ldots k$ times, which is the maximum possible $P_{V R}\left(G_{P}\right)$. 
- For some $E \in E_{P}$ if $P_{E W}(E)=0$ then $P_{E R}\left(G_{P}\right)=\Delta_{E W}-\delta_{E W}=\Delta_{E W}-0=\Delta_{E W}$

- For some $E_{i}, E_{j} \in E_{P}$ with attribute values $m_{1}, m_{2}, \ldots, m_{k}=1$ and $n_{1}, n_{2}, \ldots, n_{k}=0$ respectively, $P_{E R}\left(G_{P}\right)=1+1+\ldots k$ times, which is the maximum possible $P_{E R}\left(G_{P}\right)$.

- If $\triangle_{V W}\left(G_{P}\right)=\delta_{V W}\left(G_{P}\right)$, then $P_{V R}\left(G_{P}\right)=P_{E R}\left(G_{P}\right)=0$. Such PPFGs are referred as Zero-range PPFGs.

\section{Strong and weak P-vertices, highly strong, strong and weak P-edges in Plithogenic product fuzzy graphs}

6.1. Definition Let $G_{P}=\left(V_{P}, E_{P}\right)$ be a PPFG. Any $V \in V_{P}$ with attribute values $s_{1}, s_{2}, \ldots, s_{k}$ is said to be a strong P-vertex, if $\left(s_{1}+s_{2}+\cdots+s_{k}\right) \geq A_{V W}\left(G_{P}\right)$, where $A_{V W}\left(G_{P}\right)$ is the average P-weight of P-vertices in $G_{P}$, i.e. $A_{V W}\left(G_{P}\right)=\frac{P_{O}\left(G_{P}\right)}{\left(V_{P}\left(G_{P}\right)\right.} \cdot{ } \cdot$ Here $P_{O}\left(G_{P}\right)$ is the P-order of $G_{P}$ and $\left(V_{P}\left(G_{P}\right) \mid\right.$ is the $V_{P}$-cardinality of $G_{P}$. Otherwise, $\mathrm{V}$ is called a weak P-vertex.

6.1. Example Consider a PPFG, $G_{P}=\left(V_{P}, E_{P}\right)$ such that $V_{P}=\left\{V_{1}, V_{2}, V_{3}, V_{4}, V_{5}\right\}$ and $E_{P}=\left\{E_{1}, E_{2}, E_{3}, E_{4}, E_{5}\right\}$

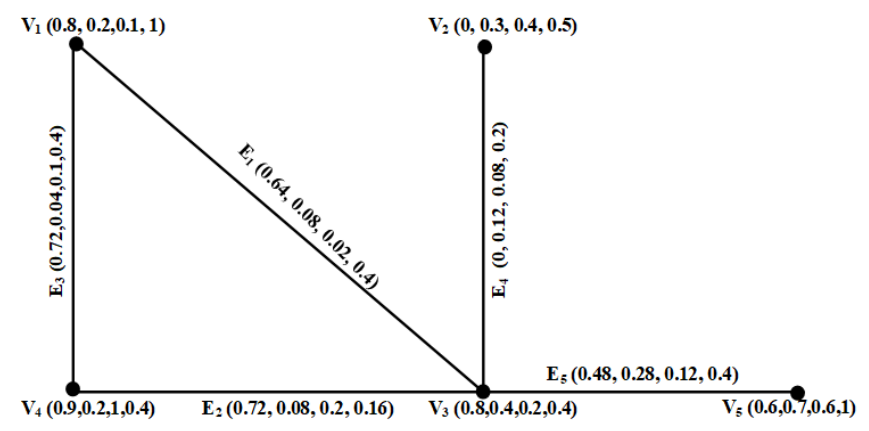

Fig 7. Strong P-vertices and Weak P- vertices

In the above PPFG, by computation we have the P-weight of each P-vertex as follows:

$P_{V W}\left(V_{1}\right)=2.1 ; P_{V W}\left(V_{2}\right)=1.2 ; P_{V W}\left(V_{3}\right)=1.8 ; P_{V W}\left(V_{4}\right)=2.5 ; P_{V W}\left(V_{5}\right)=2.9$.

Here $A_{V W}\left(G_{P}\right)=\frac{P_{O}\left(G_{P}\right)}{\left|V_{P}\left(G_{p}\right)\right|}=\frac{10.5}{5}=2.1$ Therefore, $V_{1}, V_{4}$ and $V_{5}$ are strong P-vertices. $V_{2}$ and $V_{3}$ are weak P-vertices.

6.2. Definition Let $G_{P}=\left(V_{P}, E_{P}\right)$ be a PPFG. For any $E \in E_{P}$ if its adjacent P-vertices are strong P-vertices, then $E$ is said to be a highly strong P-edge. If any one of the adjacent P-vertices of $E$ is a strong P-vertex and the other is a weak P-vertex, then $E$ is said to be a strong P-edge. If both the adjacent P-vertices of $E$ are weak P-vertices, then $E$ is said to be a weak P-edge.

6.2. Example Consider the PPFG as in Fig. 7. Here $E_{3}=V_{1} V_{4}$ is a highly strong P-edge since both $V_{1}$ and $V_{4}$ are strong P-vertices. $E_{1}=V_{1} V_{3}, E_{2}=V_{3} V_{4}$ and $E_{5}=V_{3} V_{5}$ are strong P-edges since $V_{3}$ is a weak P-vertex, and $V_{1}, V_{4}$ and $V_{5}$ are strong P-vertices. $E_{4}=V_{2} V_{3}$ is a weak P-edge since both $V_{2}$ and $V_{3}$ are weak P-vertices.

\section{Application of Plithogenic product fuzzy graph in social network}

A network is a representation of different entities and their relations or connections. A social network is a representation of a social phenomenon of a group of people, organizations, structures, events, countries, places, animals, etc. In any social network all the units not necessarily have the same importance. The density of connections at every unit is not always the same. The strength of connection between every pair of units varies due to various reasons. Therefore, all these need to be measured to decide upon the stability of any social network ${ }^{(23,24)}$. Since the property or the quality of vertices and the strength of connectedness between them are of different levels, they can be represented by fuzzy values from $[0,1]$. $^{(25,26)}$ Connectivity parameters such as number of edges present in the network, number of neighbours to each vertex, number of common neighbors, order, size, cut vertex, bridge, etc. are helpful to analyze the performance of a social network. ${ }^{(27-29)}$

Since PPFG model has four or more attribute values associated with vertices, a better prediction about the quality of Pvertices possible in PPFG model. The computation of the attribute values for the P-edges in PPFG model reduces the error to the minimum in our prediction regarding strength of connectivity. Similarly the connectivity parameters discussed in this paper are better tools to investigate connectedness in the network. In this section a new mathematical model based on PPFGs has been utilized to analyze a social network of persons. P- vertices and P-edges in the network are characterized by four attributes which have the corresponding attribute values from $[0,1]$. Some of the other terms in PPFGs and their corresponding meanings in our example of social network are as follows: 
Table 3. Terms of PPFGs and their meanings

\begin{tabular}{ll}
\hline Terms of PPFG & Meanings \\
\hline$\left|V_{P}\left(G_{p}\right)\right|$ & Total number of persons \\
$\left|E_{p}\left(G_{p}\right)\right|$ & Total number of connections \\
P-weight of a P-vertex & Strength of sociability of a person \\
P-weight of a P-edge & Strength of connectivity between two persons \\
Strong P-vertex & Important or influential person \\
Weak P-vertex & Unimportant or uninfluential person \\
Highly strong P-edge & Highly cohesive connection \\
Strong P-edge & Cohesive connection \\
Weak P-edge & Weak connection \\
$C_{P V}\left(G_{P}\right)$ & Central and significant persons of the network \\
$P_{O}\left(G_{P}\right)$ & Total strength of sociability of persons in the network \\
$P_{S}\left(G_{P}\right)$ & Total strength of connectivity in the network \\
$P_{V R}\left(G_{P}\right)$ & The maximum difference in the strengths of sociability of persons \\
$P_{E R}\left(G_{P}\right)$ & The maximum difference in the strengths of connectivity of persons \\
$d_{P}(V)$ & Strength of direct connectivity of a person \\
$t d_{P}(V)$ & Strength of direct connectivity and sociability of a person \\
$A_{V W}$ & Average strength of sociability in the network \\
Strength of a PPFP & Strength of indirect connectivity \\
$S_{C O N P}$ & Maximum strength of indirect connectivity \\
\hline
\end{tabular}

\subsection{The objectives of this analysis of the social network of persons are as follows:}

- To extract the overall impression regarding the cohesiveness of their connections.

- To find the types of relations among them in the network.

- To identify the most social, sociable, influential and central persons in the network.

- Any other relational information about the persons in the network

\subsection{Results}

Let us consider a PPFG, $G_{P}=\left(V_{P}, E_{P}\right)$ as in Fig. 8, where $V_{P}=\left\{V_{1}, V_{2}, V_{3}, V_{4}\right\}$ and $E_{P}=\left\{E_{1}, E_{2}, E_{3}, E_{4}, E_{5}, E_{6}\right\}$ are PFSs of Pvertices and relevant P-edges respectively. Here $G_{P}$ represents a social network characterized by four attributes. Here $V_{1}, V_{2}, V_{3}$ and $V_{4}$ are siblings staying in different places, while $E_{1}, E_{2}, E_{3}, E_{4}, E_{5}$ and $E_{6}$ show the existence of direct connections between them. The following matrix shows if there is a direct connection between any two persons. 1 indicates that there is a direct connection, while 0 signifies that there is no connection between a particular pair of persons.

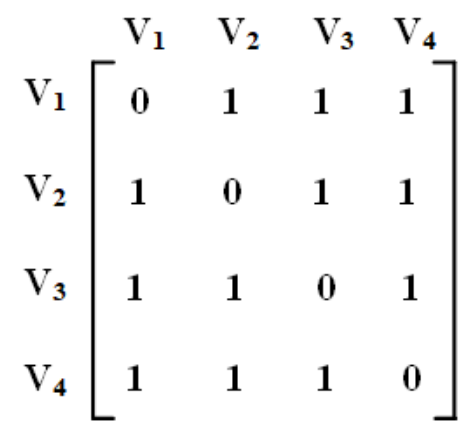

The attributes to the persons and their connections are (i) Number of visits made $\left(h_{1}\right)$, (ii) Number of gifts sent $\left(h_{2}\right)$, (iii) Number of phone calls $\left(h_{3}\right)$, and (iv) Number of WhatsApp messages $\left(h_{4}\right)$ during the period of a year. The formulae that are used to calculate the fuzzy attribute values of $h_{1}, h_{2}, h_{3}$ and $h_{4}$ for each P-vertex are as follows: 
$h_{1}$ 's fuzzy attribute value $\left(g_{1}\right)=\frac{h_{1}}{\sum_{i=1}^{4} V_{i}\left(h_{1}\right)}$

$h_{2}$ 's fuzzy attribute value $\left(g_{2}\right)=\frac{h_{2}}{\sum_{i=1}^{4} V_{i}\left(h_{2}\right)}$

$h_{3}$ 's fuzzy attribute value $\left(g_{3}\right)=\frac{h_{3}}{\sum_{i=1}^{4} V_{i}\left(h_{3}\right)}$

$h_{4}$ 's fuzzy attribute value $\left(g_{4}\right)=\frac{h_{4}}{\sum_{i=1}^{4} V_{i}\left(h_{4}\right)}$

The corresponding PPFG, $G_{P}=\left(V_{P}, E_{P}\right)$ for the above matrix and other details in Tables 4 and 5 and Table 6 are shown in Figure 8 .

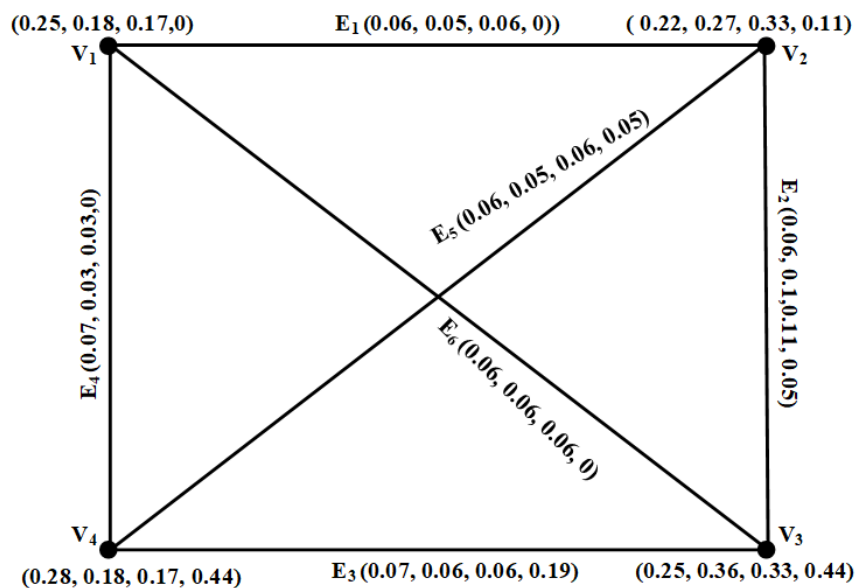

Fig 8. Social network of persons

Table 4 shows the computation of attribute values and the P-weights of P-vertices $\left(P_{V W}\right)$. All decimal values are rounded off up to two decimal places for the sake of convenience in calculation.

Table 4. Attribute values and P-weights of P-vertices

\begin{tabular}{llllllllll}
\hline Persons & $h_{1}$ & $h_{2}$ & $h_{3}$ & $h_{4}$ & $g_{1}$ & $g_{2}$ & $g_{3}$ & $g_{4}$ & $P_{V W}$ \\
\hline$V_{1}$ & 8 & 2 & 1 & 0 & 0.25 & 0.18 & 0.17 & 0 & 0.6 \\
$V_{2}$ & 7 & 3 & 2 & 1 & 0.22 & 0.27 & 0.33 & 0.11 & 0.93 \\
$V_{3}$ & 8 & 4 & 2 & 4 & 0.25 & 0.36 & 0.33 & 0.44 & 1.38 \\
$V_{4}$ & 9 & 2 & 1 & 4 & 0.28 & 0.18 & 0.17 & 0.44 & 1.07 \\
Total & 32 & 11 & 6 & 9 & 1 & 0.99 & 1 & 0.99 & 3.98 \\
\hline
\end{tabular}

The attribute values of P-edges are calculated using the usual product operator by the method shown in the definition 3.1. The attribute values of P-edges concerning $h_{1}, h_{2}, h_{3}$ and $h_{4}$, are referred by the variables $f_{1}, f_{2}, f_{3}$ and $f_{4}$ respectively. Table 5 shows the computation of attribute values and the P-weights of P-edges $\left(P_{E W}\right)$.

Table 5. Attribute values and P-weights of P-edges

\begin{tabular}{|c|c|c|c|c|c|c|}
\hline Connection & $E_{1}=V_{1} V_{2}$ & $E_{6}=V_{1} V_{3}$ & $E_{4}=V_{1} V_{4}$ & $E_{2}=V_{2} V_{3}$ & $E_{5}=V_{2} V_{4}$ & $E_{3}=V_{3} V_{4}$ \\
\hline$f_{1}$ & 0.06 & 0.06 & 0.07 & 0.06 & 0.06 & 0.07 \\
\hline$f_{2}$ & 0.05 & 0.06 & 0.03 & 0.1 & 0.05 & 0.06 \\
\hline$f_{3}$ & 0.06 & 0.06 & 0.03 & 0.11 & 0.06 & 0.06 \\
\hline$f_{4}$ & 0 & 0 & 0 & 0.05 & 0.05 & 0.19 \\
\hline$P_{E W}$ & 0.17 & 0.18 & 0.13 & 0.32 & 0.22 & 0.38 \\
\hline
\end{tabular}

Degree and total degree of P-vertices are computed and shown in Table 6.

$P_{O}\left(G_{P}\right)=3.98 \quad P_{S}\left(G_{P}\right)=1.4 \quad P_{V R}\left(G_{P}\right)=0.78 \quad P_{E R}\left(G_{P}\right)=0.25$

$$
A_{V W}\left(G_{P}\right)=\frac{P_{O}\left(G_{P}\right)}{\left(V_{P}\left(G_{P}\right)\right.}=\frac{3.98}{4}=0.995=1.00
$$


Table 6. Degree and total degree of P-vertices

\begin{tabular}{lllll}
\hline & $V_{1}$ & $V_{2}$ & $V_{3}$ & $V_{4}$ \\
\hline$d_{P}$ & 0.48 & 0.71 & 0.88 & 0.73 \\
$t d_{P}$ & 1.08 & 1.64 & 2.26 & 1.8 \\
\hline
\end{tabular}

\subsection{Discussion}

The strengths of sociability of $V_{3}$ and $V_{4}$ are greater than 1, which is the average sociability of persons in the network, while that of $V_{1}$ is the lowest, and that of $V_{2}$ is 0.93 which is slightly less than 1 . This shows that $V_{3}$ and $V_{4}$ are more sociable persons in the network. Since the strength of sociability of $V_{1}$ is the lowest, he is considered to be the least sociable among the four persons. Also the strength of connectivity between $V_{3}$ and $V_{4}$ is the best in the network. This implies that there are significant transactions and commutations taking place between $V_{3}$ and $V_{4}$. On the other hand, $V_{1} V_{4}$ has the lowest strength of connectivity as its $\mathrm{P}$-weight is the least in the network. This indicates that $V_{1}$ 's relationship with $V_{4}$ is the weakest in the network. However, the strengths of connectivity of $V_{1}$ with $V_{2}$ and $V_{3}$ are satisfactory.

The degree and the total degree of $V_{3}$ are higher than those of others in the network. Therefore, both in strength of direct connectivity as well as in strength of sociability $V_{3}$ is the best. Hence $V_{3}$ is more significant and influential for the overall interactions, flow of information and the smooth functioning of the network. $V_{3}$ is a leader inside this network ${ }^{(24)}$.

The overall impression regarding the strength of sociability of persons in the network seems to be very good as three out of four persons have better strengths of sociability. Since the grading of strength of connectivity among them depends on their strengths of sociability, based on the data in Table 4 we conclude that $E_{3}$ is a highly cohesive connection, $E_{2}, E_{4}, E_{5}$ and $E_{6}$ are cohesive connections, and $E_{1}$ is a weak connection.

\section{Conclusion}

PPFGs has been newly defined and its properties are investigated with results and examples in this paper. It is proved that every PPFG is a PFG, but the converse need not be true. It is also verified that every subgraph of a PPFG is PPFG. Finally, the utility of PPFG and its application in a small-sized social network is demonstrated to understand the advantage of it in decision making process. As future work, A large-scale practical network can be modeled using PPFG to analyze a real-life problem under uncertain environment. Operations on PPFGs are also areas of scope for future research to understand better the nature of PPFGs.

\section{References}

1) Zadeh LA. Fuzzy sets. Information and Control. 1965;8(3):338-353. Available from: https://dx.doi.org/10.1016/s0019-9958(65)90241-x.

2) Mythili V, Kaliyappan M, Hariharan S. A Review of Fuzzy Graph Theory. International Journal of Pure and Applied Mathematics. 2017;113(12):187-195. Available from: https://acadpubl.eu/jsi/2017-113-pp/articles/12/21.pdf.

3) Rosenfeld A. Fuzzy Graphs. In: Fuzzy Sets and their Applications to Cognitive and Decision Processes. Academic Press. 1975;p. 77-95. Available from: https://doi.org/10.1016/B978-0-12-775260-0.50008-6.

4) Atanassov KT. Intuitionistic Fuzzy Sets. In: Fuzzy Sets and Systems. 1986;p. 87-96. Available from: https://doi.org/10.1016/S0165-0114(86)80034-3.

5) Parvathi R, Karunambigai MG. Intuitionistic Fuzzy Graphs. In: Computational Intelligence, Theory and Applications. Springer Berlin Heidelberg. 2006;p. 139-150. Available from: https://doi.org/10.1007/3-540-34783-6_15.

6) Zuo C, Pal A, Dey A. New Concepts of Picture Fuzzy Graphs with Application. Mathematics. 2019;7(5):470. Available from: https://dx.doi.org/10.3390/ math7050470.

7) Shi X, Kosari S. Certain Properties of Domination in Product Vague Graphs With an Application in Medicine. Frontiers in Physics. 2021;9:385. Available from: https://dx.doi.org/10.3389/fphy.2021.680634.

8) Rosyida I, Suryono S. Coloring picture fuzzy graphs through their cuts and its computation. International Journal of Advances in Intelligent Informatics. 2021;7(1):63-75. Available from: https://dx.doi.org/10.26555/ijain.v7i1.612.

9) Bhattacharya A, Pal M. Fuzzy covering problem of fuzzy graphs and its application to investigate the Indian economy in new normal. Journal of Applied Mathematics and Computing. 2021;p. 1-32. Available from: https://dx.doi.org/10.1007/s12190-021-01539-4.

10) Smarandache F. Neutrosophic set - a generalization of the intuitionistic fuzzy set. IEEE. 2006;p. 38-42. Available from: https://doi.org/10.1109/GRC. 2006.1635754

11) Vasantha WB, Ilanthenral K, Smarandache K, F. Neutrosophic Graphs : A New Dimention to Graph Theory Brussels: EuropaNova. 2015. Available from: https://core.ac.uk/download/pdf/270100476.pdf.

12) Smarandache F. Refined literal indeterminacy and the multiplication law of sub-indeterminacies. Infinite Study. 2015. Available from: https://citeseerx. ist.psu.edu/viewdoc/download?doi=10.1.1.962.626\&rep=rep1\&type=pdf $\#$ page $=60$.

13) Broumi S, Talea M, Bakali A, Smarandache F. Single Valued Neutrosophic Graphs. Journal of New Theory. 2016;(10):86-101. Available from: https://dergipark.org.tr/en/download/article-file/407546.

14) Smarandache F. Plithogeny, Plithogenic Set, Logic, Probability, and Statistics Brussels, Belgium. Pons Editions. 2017. Available from: https:// digitalrepository.unm.edu/math_fsp/20. 
15) Vasantha WB, Ilanthenral K, Smarandache F. Plithogenic Graphs Bruxelles;vol. EuropaNova: 2020. . Available from: https://www.researchgate.net/ publication/343769158_Plithogenic_Graphs.

16) Yeh RT, Bang SY. Fuzzy relations, fuzzy graphs, and their applications to clustering analysis. In: Fuzzy sets and their applications to Cognitive and Decision Processes. Academic Press. 1975;p. 125-149.

17) Gani AN, Ahamed MB. Order and size in fuzzy graphs. Bulletin of Pure and Applied Sciences. 2003;22(1):145-148.

18) Rashmanlou H, Samanta S, Pal M, Borzooei RA. Product of bipolar fuzzy graphs and their degree. International Journal of General Systems. 2016;45(1):114. Available from: https://dx.doi.org/10.1080/03081079.2015.1072521.

19) Pal A, Samanta S, Pal M. Concept of fuzzy planar graphs. In: Science and Information Conference. IEEE. 2013;p. 557-563. Available from: https://ieeexplore.ieee.org/abstract/document/6661793.

20) Samanta S, Pal M. Fuzzy k-Competition Graphs and p-Competition Fuzzy Graphs. Fuzzy Information and Engineering. 2013;5(2):191-204. Available from: https://dx.doi.org/10.1007/s12543-013-0140-6.

21) Samanta S, Pal M. Fuzzy Planar Graphs. IEEE Transactions on Fuzzy Systems. 2015;23(6):1936-1942. Available from: https://dx.doi.org/10.1109/tfuzz. 2014.2387875.

22) Al-Hawary T, Horani B. On Product Fuzzy Graphs. Annals of Fuzzy Mathematics and Informatics. 2016;12(2):279-294.

23) Samanta S, Pal M. A new approach to social networks based on fuzzy graphs. Turkish Journal of Fuzzy Systems. 2014;5(2):78-99.

24) Casasús-Estellés T, Yager RR. Fuzzy Concepts in Small Worlds and the Identification of Leaders in Social Networks. Information Processing and Management of Uncertainty in Knowledge-Based Systems. 2014;p. 37-45.

25) Binu M, Mathew S, Mordeson JN. Connectivity status of fuzzy graphs. Information Sciences. 2021;573:382-395. Available from: https://dx.doi.org/10. 1016/j.ins.2021.05.068.

26) Naeem T, Gumaei A, Jamil MK, Alsanad A, Ullah K. Connectivity Indices of Intuitionistic Fuzzy Graphs and Their Applications in Internet Routing and Transport Network Flow. Mathematical Problems in Engineering. 2021;2021:1-16. Available from: https://dx.doi.org/10.1155/2021/4156879.

27) Mahapatra R, Samanta S, Pal M, Xin Q. Link Prediction in Social Networks by Neutrosophic Graph. International Journal of Computational Intelligence Systems. 2020;13(1):1699-1713. Available from: https://dx.doi.org/10.2991/ijcis.d.201015.002.

28) Khan WA, Ali B, Taouti A. Bipolar Picture Fuzzy Graphs with Application. Symmetry. 2021;13(8 (1427)):1-22. Available from: https://dx.doi.org/10. $3390 /$ sym 13081427.

29) Sunita MS, Mathew S. Fuzzy Graph Theory: A survey. Annals of Pure and Applied Mathematics. 2013;4(1):92-110. 